\title{
Embolización intraprostática de cristales de colesterol
}

\author{
J. Planas Morin, F. Aguiló Lucía, F.J. Suárez Novo, F.J. Elias Cañavera, N. Serrallach i Milá \\ Servicio de Urología. Hospital Universitario de Bellvitge. Hospitalet de Llobregat. Barcelona.
}

Actas Urol Esp 2005; 29 (1): 105-106

\section{RESUMEN}

EMBOLIZACIÓN INTRAPROSTÁTICA DE CRISTALES DE COLESTEROL

La Enfermedad por émbolos de colesterol (EEC) es una patología poco conocida pero con una alta mortalidad asociada. La presencia de embolias de cristales de colesterol a nivel intraprostático como hallazgo poco común en las biopsias prostáticas transrectales en un enfermo con factores de riesgo tromboembólico, debe alertarnos sobre la posible existencia de la EEC.

Palabras clave: Enfermedad por émbolos de colesterol. Próstata. Biopsias prostáticas transrectales.

\section{ABSTRACT \\ INTRAPROSTATIC EMBOLIZATION OF CHOLESTEROL CRYSTALS}

The cholesterol embolism syndrome (CES) is an unusual disease that carries a high mortality rate. Finding intraprostatic cholesterol crystal embolization as the result of transrectal prostate biopsy in a patient with several risk factors for atherosclerosis, should alert the urologist to the possibility of CES existence.

Key words: Cholesterol embolism syndrome. Prostate. Transrectal prostate biopsy.

$\mathrm{P}$ resentamos a continuación el caso de un paciente varón de 62 años de edad, fumador de 20 cigarrillos/día, con antecedentes de Diabetes Mellitus no insulinodependiente, herniorrafia inguinal derecha y proteinuria estudiada y controlada por el Servicio de Nefrología, que es remitido a nuestro Servicio por su médico de cabecera por el hallazgo analítico de PSA de $12.5 \mathrm{ng} / \mathrm{ml}$ en julio de 2002.

A la exploración física destaca una próstata de tamaño grande, plana, de consistencia adenomatosa, bien delimitada y sin nódulos pétreos. Abdomen blando y depresible sin masas ni visceromegalias. Pene y testículos sin alteraciones.

Ante el PSA de $12.5 \mathrm{ng} / \mathrm{ml}$ y la sospecha bioquímica de carcinoma prostático, practicamos ecografia transrectal y biopsias prostáticas por sextantes: próstata con un peso estimado de 42 gramos. Se practican biopsias prostáticas obteniendo 3 cilindros del lóbulo derecho y 3 cilindros del lóbulo izquierdo. Dictamen anatomopatológico: embolias de cristales de colesterol en todos los cilindros remitidos. Hiperplasia nodular.

Ante la sorpresa por el hallazgo anatomopatológico, hemos realizado una revisión de la literatura encontrando muy pocos casos descritos, recomendándose descartar enfermedad ateroembólica. Realizamos consulta con el Servicio de Cirugía Vascular que no encontró signos ni síntomas de isquemia en la exploración física del paciente. Se practicó Ecodoppler del eje aortoilíaco objetivando placas de ateroma en aorta terminal y en ambas arterias iliacas no ulceradas ni estenóticas. Dada la ausencia de clínica isquémica y dados los hallazgos ecográficos vasculares que no sugieren actuación terapéutica actualmente, el paciente fue dado de alta por parte del Servicio de Cirugía Vascular.

Actualmente el paciente sigue controles por nuestro Servicio. El valor actual de PSA (enero 2003) es de $16.6 \mathrm{ng} / \mathrm{ml}$, sin cambios en la exploración rectal y sin signos ni síntomas vasculares. Hemos adoptado una conducta expectante. 


\section{DISCUSION}

El hallazgo y la incidencia de embolia de cristales de colesterol en las biopsias prostáticas es infrecuente y no bien conocida (en estudios postmorten se ha objetivado en $2.5 \%$ de enfermos con enfermedad ateroembólica). El primero en describirlas fue Zac en $1949^{1,2}$. Este hallazgo puede estar relacionado con la Enfermedad por émbolos de colesterol. Esta enfermedad se caracteriza por la oclusión de vasos de pequeño calibre por microcristales de colesterol que se desprenden de las placas de ateroma de las grandes arterias. Su incidencia se desconoce ya que sus manifestaciones clínicas son poco específicas o porque en ocasiones puede cursar de forma asintomática ${ }^{3}$.

Los órganos afectados con mayor frecuencia son la piel, el riñón y el sistema nervioso central, pudiéndose afectar cualquier otro órgano. Los factores desencadenantes más frecuentemente relacionados con esta enfermedad son los procedimientos quirúrgicos vasculares y el tratamiento anticoagulante, si bien se trata de un fenómeno que también puede darse de forma espontánea. Los pacientes afectos de esta enfermedad suelen presentar dos o más factores de riesgo aterosclerótico (hipertensión, tabaquismo, diabetes mellitus, dislipemia).

La tríada clínica más sugestiva del cuadro es la aparición de lesiones cutáneas asociadas a hipertensión arterial e insuficiencia renal aguda. Las manifestaciones clínicas más habituales son la livedo reticularis y el hallazgo de lesiones purpúricas, si bien también pueden presentarse en forma de lesiones isquémicas distales, úlceras o gangrena que precisan en ocasiones de amputación.

El diagnóstico suele realizarse por técnica de biopsia cutánea donde se objetiva la presencia de cristales de colesterol en el interior de los vasos localizados en el límite dermo-hipodérmico. Los hallazgos analíticos son poco específicos $\mathrm{y}$ de escaso interés para establecer el diagnóstico ${ }^{3,4}$.
La mortalidad es alta (75-80\%) siendo la afectación renal el principal factor pronóstico. No existe un tratamiento efectivo, y la actitud a seguir cuando se realiza el diagnóstico debe dirigirse a eliminar de forma precoz el factor desencadenante y tratar las complicaciones secundarias. $\mathrm{El}$ diagnóstico precoz y la consiguiente retirada del factor desencadenante puede reducir la mortalidad hasta situarla en el $12 \%$.

A pesar de que nuestro paciente no presenta en la actualidad clínica ni afectación vascular severa, sí cumple dos criterios de riesgo tromboembólico (tabaquismo y diabetes mellitus).

Pensamos que el hallazgo de embolia de cristales de colesterol a nivel intraprostático en las biopsias prostáticas transrectales realizadas para descartar el carcinoma de próstata en un paciente con factores de riesgo tromboembólico, debe poner en alerta al urólogo de cara a realizar un diagnóstico precoz de la Enfermedad por émbolos de colesterol (enfermedad poco conocida y con una elevada tasa de complicaciones y alta mortalidad) para reducir las graves secuelas que ésta puede conllevar.

\section{REFERENCIAS}

1. Zac, F.G. and Elias K. Embolisation with material form atheromata. Am. J. Med. Sci. 1949, 218: 510-515.

2. Adamson A.S. and Snell M.E. Atheroembolism of the prostate. Br J Urol. 1993 Aug;72(2):254.

3. Ribera M., Bigata X., Fuentes M.J.,Bielsa I., Ferrándiz C. Enfermedad por émbolos de colesterol: estudio de 16 casos. Rev Clin Esp. 2000 Dec;200(12):659-63.

4. Knechtges T., Defever B. Cholesterol emboli in transurethral curettings: report of 4 cases. J Urol.1975 July, 114: 102106.

J. Planas Morín

Crta de Moja $\mathrm{n}^{\circ} 12,2^{\circ} 3^{\circ}$

Vilafranca del Penedès

08720. Barcelona

(Trabajo recibido el 17 marzo de 2004) 